\title{
Posterior Reversible Encephalopathy Syndrome (PRES) (Report of three cases with brief review of literature)
}

\author{
Dr. K.M.Raul ${ }^{1}$, Dr.Mahendra Wawhal ${ }^{2}$, Dr.sharad Garudkar ${ }^{3}$, Dr.Vajed Mogal ${ }^{4}$ \\ ${ }^{1}$ Professor, ${ }^{2}$ Associate Professor, ${ }^{3}$ Chief Resident In Medicine, ${ }^{4}$ Senior Resident In Medicine, \\ Department of Medicine, Mahatma Gandhi Mission's Medical College \& Hospital, CIDCO, N-6, Aurangabad, \\ Maharashtra., India - 431003
}

\begin{abstract}
We report three cases of Posterior Reversible Encephalopathy Syndrome in different settings. First case was associated with systemic lupus erythematosus with gestational hypertension and lupus nephritis. Second case had no history of hypertension while third patient had only transient rise of blood pressure during \& soon after an episode of seizures. All three cases had complete recovery. Posterior Reversible Encephalopathy Syndrome is a clinical-neuroradiological term, often associated with delayed postpartum eclampsia \& classical imaging features usually in the form of bilateral, symmetrical, reversible white matter oedema involving commonly the posterior cerebral circulation. Diagnosing these lesions early \& providing appropriate treatment is important to achieve an optimal neurological outcome.
\end{abstract}

Submitted Date 29 June 2013

Accepted Date: 04 July 2013

\section{Introduction}

Most cases of eclampsia usually occurs from 20 weeks of gestation up to first 2 days after delivery ${ }^{1}$. Some cases of eclampsia have been reported as late as 23 days postpartum ${ }^{2}$. In classical case of eclampsia, the pre-eclamptic prodrome of proteinuria and hypertension precedes the onset of generalised seizure ${ }^{1}$.

In contrast, Delayed postpartum eclampsia is characterised by the onset of generalized seizures in a puerperal women from 2 days up to 30 days postpartum, frequently in a patient who had a normal pregnancy and delivery and without pre-eclamptic prodrome of proteinuria \& hypertension ${ }^{3,4}$. Posterior Reversible Encephalopathy Syndrome is an obstetric emergency frequently occurring in a postpartum period.

Case 1:

\section{Case History}

A 25 years old patient gravida 3,para 1, living 1, abortion 1 had history of 9 months amenorrhea was admitted with chief complaints of swelling over the both feet since 2 months and oral ulcers since last 20 to 25 days. Patient was a diagnosed case of Systemic Lupus Erythematosus (SLE) with Gestational Hypertension since 6 months. On physical examination, the patient was afebrile with tachycardia of 104/min, blood pressure was 160/100 $\mathrm{mmHg}$, bilateral pitting pedal oedema was present. Urine dipstick showed 4+ albuminuria.

In view of high risk pregnancy (previous Lower Segment Caesarean Section, Gestational hypertension \& Lupus Nephritis), patient was taken for elective LSCS. LSCS was uneventful with delivery of healthy baby. On $12^{\text {th }}$ day postpartum patient experienced intense headache, nausea, vomiting and blurring of vision, followed by generalized tonic-clonic seizures. The aetiology of seizures was unclear initially. The differential diagnosis included subarachnoid haemorrhage, space occupying lesion in brain, intracranial haemorrhage, ischemic stroke, venous thrombosis, HELLP syndrome, Meningo-encephalitis.

Computed Tomography (CT) Scan of brain revealed subcortical white matter hypodensities in bilateral occipital lobes, mostly representing vasogenic oedema suggest possibilities of posterior reversible encephalopathy. MRI and MRV were ordered to rule out thrombosis and space occupying lesion. MRI scan of brain showed gyral oedema in bilateral frontal and parieto-occipital parenchyma suggest possibilities of reversible encephalopathy syndrome. MRV was normal. The diagnosis of PRES was confirmed by imaging and clinical course as well as by ruling out other aetiologies. Patient given antihypertensives and magnesium sulphate and anti-epileptic. The patient had no further seizures, recovered slowly and was discharged on hospital day 40 .

Case 2:

A 23 years old patient gravida1, para 1 had normal pregnancy and normal full term vaginal delivery of healthy baby and discharged from hospital on $3^{\text {rd }}$ day postpartum. On $4^{\text {th }}$ day postpartum patient experienced mild headache, nausea and blurred vision in morning hours to which patient neglected. On the same day 
afternoon patient developed generalised tonic-clonic seizures for which she was admitted. On physical examination patient was afebrile, blood pressure was $130 / 80 \mathrm{mmHg}$. Patient's serial blood pressure recordings were within normal limit. Urine dipstick showed no albuminuria. Patients all the laboratory investigations and metabolic profile were within normal limit. Patients MRI brain with MRV \& MRA were ordered which showed areas of altered signal seen in both occipito-parietal and frontal regions dominantly involving the junctional zone of cortex and white matter with adjacent focal oedema and early ischemia seen suggest possibility of posterior reversible encephalopathy. MR Angiography \& venography revealed normal report. Patient given magnesium sulfate and improved.

Case 3:

A 24 years old patient primigravida had normal pregnancy, underwent LSCS in view of prolonged labour and foetal distress. LSCS was uneventful with delivery of healthy baby. On $6^{\text {th }}$ postoperative day patient developed intense headache, vomiting followed by generalised tonic-clonic seizures. At the time of seizures \& after short period of seizures her blood pressure was 170/100. Her ANC follow up record showed that patient was previously healthy and normotensive. Her haemogram, serum uric acid, urine examination \& fundus examination was normal. MRI Brain showed bilateral symmetric fronto-parietal cortical abnormalities with mild swelling, results consistent with PRES. MRA \& MRV were normal. Patient given antihypertensive for short period and patient was discharged on hospital day 12.

\section{Discussion}

PRES was first described by Hinchey \& colleagues in 1996 as Reversible Posterior Leukoencephalopathy Syndrome. PRES has been described as various non-specific symptoms of intense headache, visual problems (e.g. blurring of vision, hemianopia, cortical blindness, hemineglect), altered consciousness and generalised seizures. Rarely the patients may develop focal neurological deficits like paresis.

PRES may develop suddenly or over several days ${ }^{5}$. The term PRES is a misnomer as it occurs not always in posterior cerebral circulation but may occur primarily in anterior cerebral circulation. Also, PRES is not always reversible; it can be irreversible if the aetiology is not treated. In addition, patient in PRES may not always present with an encephalopathy, instead patient may have various nonspecific clinical features other than encephalopathy. In PRES white matter is mostly involved but it may involve deep white and gray matters ${ }^{6}$.

\section{Risk factors:}

PRES a recently described clinical-neuroradiological term that is associated with several medical condition besides preeclampsia/eclampsia and hypertension e.g. Renal failure, Post-transplantation (Allogeneic bone marrow transplantation; Solid organ transplantation), Immunosuppressive therapy (Cyclosporine; Tacrolimus) Autoimmune diseases (SLE; Systemic Sclerosis; Wegener's Granulomatosis; Poly Arteritis Nodosa), Post-cancer chemotherapy and has recently shown to be associated with infection, sepsis, and shock ${ }^{7}$.

\section{Radiological findings of PRES:}

The characteristic imaging in PRES is cortical or subcortical areas of hypoattenuation with a predominantly bilateral, symmetrical, posterior distribution in the parietal \& occipital white matter on CT or T2 hyperintensity on MR imaging ${ }^{8}$. On MR imaging, the findings are most apparent on fluid- attenuated inversion recovery (FLAIR) images. Most evident on T2-weighted MRI images, the lesions are hyperintense and located at the gray-white junction. The parietal and occipital lobes are most commonly affected, followed by in descending order the frontal lobes, the inferior temporal-occipital junction, and the cerebellum ${ }^{5}$. Involvement of the basal ganglia, brain stem, and deep white matter including the splenium of corpus callosum can also be seen'. Frequent involvement of posterior cerebral circulation is not well understood. It is presumed that intracranial arterioles in anterior circulation have extensive sympathetic innervations than in posterior circulation which are responsible for protection from raised blood pressure. This shows that the amount of sympathetic innervation and the degree of parenchymal involvement are inversely proportional in PRES.

However, in some cases the protective effect of sympathetic innervation in anterior circulation can be overcome and anterior circulation can be involved called as Atypical PRES ${ }^{\mathbf{1 0}}$. McKinny \& colleagues reported that PRES can be unilateral. This atypical unilateral involvement can cause difficulty in the diagnosis of PRES. Follow up neuroimaging studies in PRES shows nearly complete resolution of imaging findings, and suggest that PRES is usually associated with reversible cerebral oedema without infarction \& confirms the diagnosis ${ }^{11}$.

\section{Pathophysiology of PRES:}

The pathophysiology of PRES is not well understood. Vasogenic theory i.e. hypertension with loss of autoregulation remains a widely accepted consideration for the development of brain oedema ${ }^{\mathbf{1 2}}$. Sometimes patients with normal blood pressure may develop PRES if they have substantial rise in blood pressure which is considered to be within the range of normal blood pressure. This is believed to be due to some neurotoxic 
substances ${ }^{13}$. PRES is seen in the absence of hypertension in $20 \%-40 \%$ of patients ${ }^{5}$. Alternatively, endothelial dysfunction/injury, hypoperfusion, and vasoconstriction may lead to altered integrity of the blood-brain barrier $^{14}$. In the latter, though some degree of hypertension is present, reported blood pressure usually do not reach the limit of autoregulation (mean arterial pressure $>160 \mathrm{~mm} \mathrm{Hg}$ ).

\section{Treatment:}

The objective of the treatment of PRES is directed at the underlying aetiology e.g., control of blood pressure, reducing the dose or withholding the offending drug in patients undergoing chemotherapy or immunosuppression. Some physicians use antiepileptic drugs or Magnesium sulphate to avoid the progress of seizures, but their role is controversial or yet to be established.

\section{Prognosis:}

The clinical outcome is variable but is mostly favourable with prompt treatment of the underlying cause and repeat neuroimaging may not be necessary. Immediate action to identify potential triggering drugs, controlling hypertension, and treating aetiology of PRES can lead to complete reversal of radiological and neurological findings ${ }^{15}$. However, in few patients, PRES progresses to ischemia, infarction, or death.

\section{Conclusion}

The pathophysiology responsible for the PRES remains controversial. PRES is usually reversible with early treatment of the aetiology responsible for it otherwise it has been shown to progress from reversible cerebral oedema to irreversible ischemic changes if appropriate treatment not started early ${ }^{16}$. Early diagnosis and treatment is essential to ensure the best possible outcome. The occurrence of delayed postpartum eclampsia without typical preeclamptic prodrome of proteinuria \& hypertension emphasizes the need of awareness of the diagnosis of PRES. Patients should be routinely counselled about the warning signs of PRES during postpartum period \& on hospital discharge.

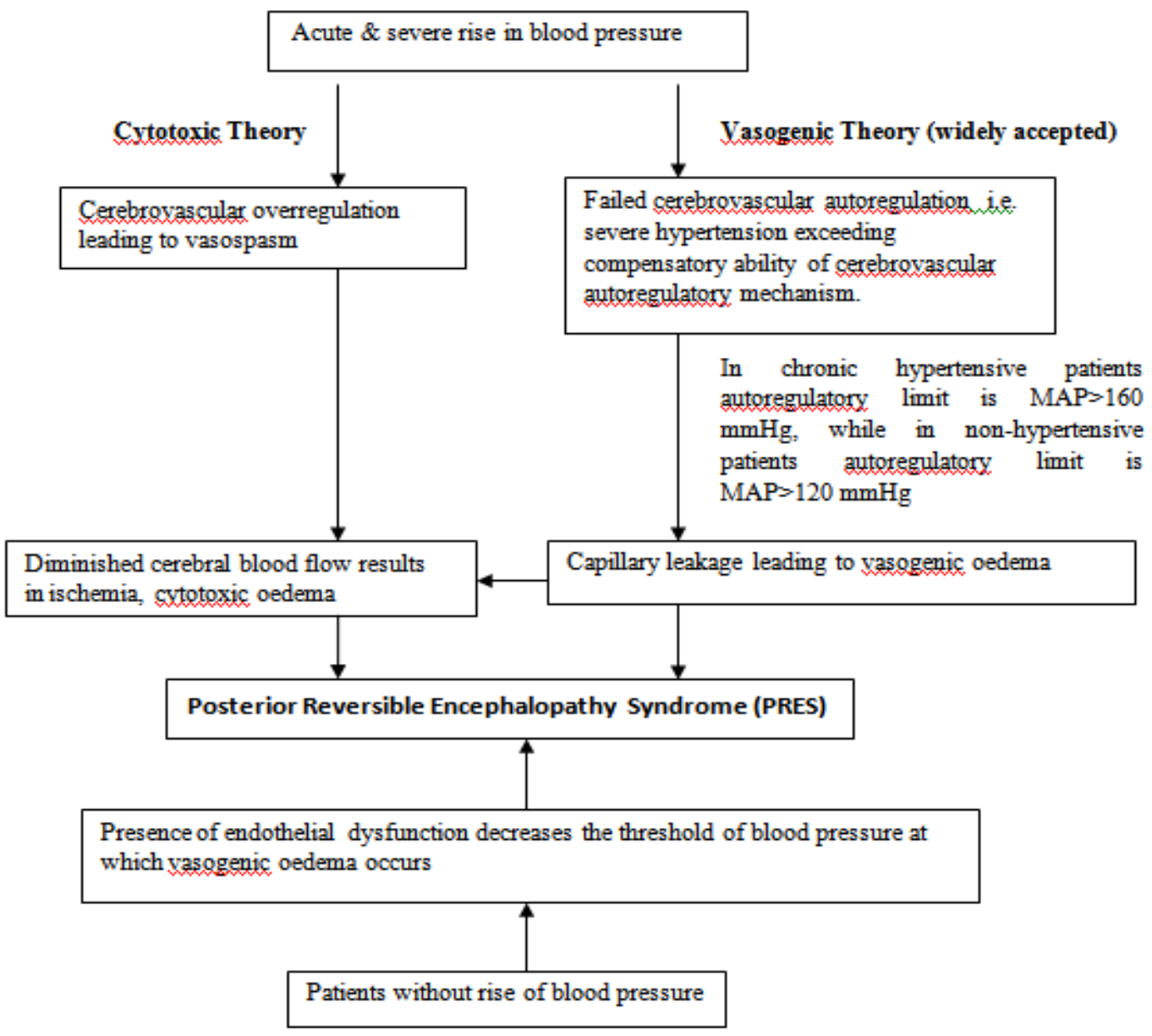

Fig. 1 Pathophysiology of PRES ${ }^{1}$ 


\section{REFERENCES}

[1] Williams Obstetrics book, $23^{\text {rd }}$ edition, Pregnancy Hypertension, page 708-709.

[2] Sibai bm. Diagnosis prevention and management of eclampsia, Obstet Gynecol. 2005 Feb; 105(2):402-10.

[3] Lubarsky SL, Barton JR, Friedman SA, Nasreddine S,Ramaddan MK, Sibai BM, Late postpartum eclampsia revisited. Obstet Gynecol 1994;83:502-5.

[4] Felz MW, Barnes DB, Figueroa RE. Late postpartum eclampsia 16 days after delivery: case report with clinical,radiologic, and pathophysiologic correlations. J Am BoardFam Pract 2000;13:39-46

[5] Bartynski WS. Posterior reversible encephalopathy syndrome, part 1: fundamental imaging and clinical features, AJNR 2008;29(6):1036-42.

[6] V. L. Stott, M. A. Hurrell, T. J. Anderson, Reversible posterior leukoencephalopathy syndrome: a misnomer reviewed, Internal Medicine Journal 2005; 35: 83-90

[7] Bartynski WS, Boardman JF, Zeigler ZR, et al, Posterior reversible encephalopathy syndrome in infection, sepsis and shock. AJNR 2006; 27:2179-90

[8] Hinchey J, Chaves C, Appignani B, Breen J, Pao L, Wang A, Pessin MS, Lamy C, Mas JL, Caplan LR, A reversible posterior leukoencephalopathy syndrome, NEJM, 1996 Feb 22; 334(8):494-500.

[9] W.S. Bartynskia and J.F. Boardmana, Distinct Imaging Patterns and Lesion Distribution in Posterior Reversible Encephalopathy Syndrome, AJNR, August 2007; 28: 1320-1327

[10] Silvia Pugliese, V. Finocchi, M. L. Borgia, C. Nania, B. Della Vella, A. Pierallini, A. Bozzao, Intracranial hypotension and PRES: case report, J Headache Pain (2010) 11:437-440

[11] A Primavera, D Audenino, N Mavilio, L Cocito, Reversible posterior leucoencephalopathy syndrome in systemic lupus and vasculitis, Ann Rheum Dis 2001;60:534-537

[12] Strandgaard S, Olesen J, Skinhoj E, et al. Autoregulation of brain circulation in severe arterial hypertension. BMJ 1973; 1:507-10

[13] Mueller-Mang C, Mang T, Pirker A, Klein K, Prchla C, Prayer D, Posterior reversible encephalopathy syndrome: do predisposing risk factors make a difference in MRI appearance?, Neuroradiology. 2009 Jun;51(6):373-83

[14] B L Trommer, D Homer and M A Mikhael, Cerebral vasospasm and eclampsia, Stroke American Heart Association,1988; 19:326329

[15] Jason K Kur and John M Esdaile Posterior reversible encephalopathy syndrome--an underrecognized manifestation of systemic lupus erythematosus, The Journal of Rheumatology vol. 33 no. 11 2178-2183

[16] Coughlin WF, McMurdo SK, Reeves T, MR imaging of postpartum cortical blindness, J Comput Assist Tomogr 1989; 13:572-76 